\title{
International multicenter comparative trial of endoscopic ultrasonography-guided gastroenterostomy versus surgical gastrojejunostomy for the treatment of malignant gastric outlet obstruction
}

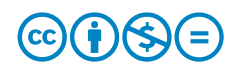

\author{
Authors \\ Mouen A. Khashab ${ }^{1}$, Majidah Bukhari' ${ }^{1}$, Todd H. Baron², Jose Nieto ${ }^{3}$, \\ Mohamad El Zein ${ }^{1}$, Yen-I Chen ${ }^{1}$, Yamile Haito Chavez ${ }^{1}$, Saowanee \\ Ngamruengphong ${ }^{1}$, Ahmad S. Alawad ${ }^{1}$, Vivek Kumbhari ${ }^{1}$, Takao \\ Itoi $^{4}$ \\ Institutions \\ 1 Division of Gastroenterology and Hepatology, Johns Hopkins \\ Medical Institutions, Baltimore, Maryland, United States \\ 2 Division of Gastroenterology and Hepatology, University of \\ North Carolina, Chapel Hill, North Carolina, United States \\ 3 Borland-Groover Clinic, Jacksonville, Florida, United States \\ 4 Division of Gastroenterology and Hepatology, Tokyo Medical \\ University, Shinjuku-ku, Tokyo, Japan
}

submitted: 10.9 .2016

accepted after revision: 30.12 .2016

Bibliography

DOI http://dx.doi.org/10.1055/s-0043-101695 |

Endoscopy International Open 2017; 05: E275-E281

(c) Georg Thieme Verlag KG Stuttgart · New York

ISSN 2364-3722

Corresponding author

Takao Itoi, Division of gastroenterology and hepatology, Tokyo

Medical University, Shinjuku-ku, Tokyo, Japan

itoi@tokyo-med.ac.jp

\begin{abstract}
Background and study aims EUS-guided gastroenterostomy (EUS$\mathrm{GE}$ ) is a novel procedure that potentially offers long-lasting luminal patency without the risk of tumor ingrowth/overgrowth. This study compared the clinical success, technical success, adverse events (AEs), length of hospital stay (LOHS) and symptom recurrence in EUS-GE versus SG].

Methods This was a multicenter international retrospective comparative study of EUS-GE and SG] in patients with malignant gastric outlet obstruction (GOO) who underwent either EUS-GE or SGJ. EUS-GE was performed using lumen apposing metal stents.

Results A total of 93 patients with malignant $\mathrm{GOO}$ treated with either EUS-GE $(n=30)$ or SGJ $(n=63)$ were identified. Peritoneal carcinomatosis was present in $13(43 \%)$ patients in the EUS-GE group and $7(11 \%)$ patients in the SGJ group $(P<0.001)$. Although the technical success rate was significantly higher in the SG] group as compared to the EUS-GE group ( $100 \%$ vs. $87 \%, P=0.009$ ), the clinical success rate was not different ( $90 \%$ vs. $87 \%, P=0.18$, OR $0.8,95 \% \mathrm{Cl} 0.44-7.07)$. The rate of AEs was lower in the EUS-GE group, but the difference was not statistically significant ( $16 \%$ vs $25 \%, P=0.3)$. The mean LOHS was similar in the EUS-GE group compared to SGJ $(P=0.35)$. The rate of recurrent GOO was not different between the two groups ( $3 \%$ vs. $14 \%, P=0.08$ ). Similarly, the mean time to reintervention was similar ( 88 days vs. 121 days, $P=0.83$ ).

Conclusions EUS-GE is associated with equivalent efficacy and safety as compared to surgical GJ. This is the first comparative trial between both techniques and suggests EUS-GE as a non-inferior but less invasive alter to surgery.
\end{abstract}

\section{Introduction}

Patients with malignant periampullary tumors frequently present with gastric outlet obstruction (GOO) [1]. Most of these patients have advanced, non-resectable tumors and thus, symptom palliation and improving quality of life are the usual goals of any intervention [1]. Some patients are candidates for neoadjuvant chemoradiation therapy. Palliation of GOO symptoms and maintaining adequate nutritional status are crucial in this difficult-to-treat population. The current most common interventions performed in such patients for the treatment of $\mathrm{GOO}$ are placement of enteral self-expandable metallic stents (SEMS) or surgical gastrojejunostomy (SG]) [2,3]. Three randomized trials have compared outcomes of both approaches with mixed results [4-6]. The main shortcoming of enteral SEMS placement is recurrent GOO due to tumor ingrowth/over- growth, which occurs in the majority of patients who survive longer than 6 months [6]. On the other hand, the main limitation of SG] is its invasive nature, especially in such patients with advanced malignancies and poor nutritional status. In addition, SG] is associated with frequent complications, such as perioperative infections and gastroparesis [7].

EUS-guided gastroenterostomy (EUS-GE) is the latest breakthrough technique for management of patients with benign and malignant GOO $[8,9]$. EUS provides ready access to small bowel loops distal to the site of obstruction through a transgastric approach. With the recent availability of lumen-apposing metal stents (LAMS) [10], creation of an endoscopic GE under EUS guidance became feasible [11]. Several case reports and case series demonstrated the feasibility, efficacy and safety of EUS-GE $[9,11-13]$. However, there currently exist no studies comparing EUS-GE to the other currently practiced treatment 

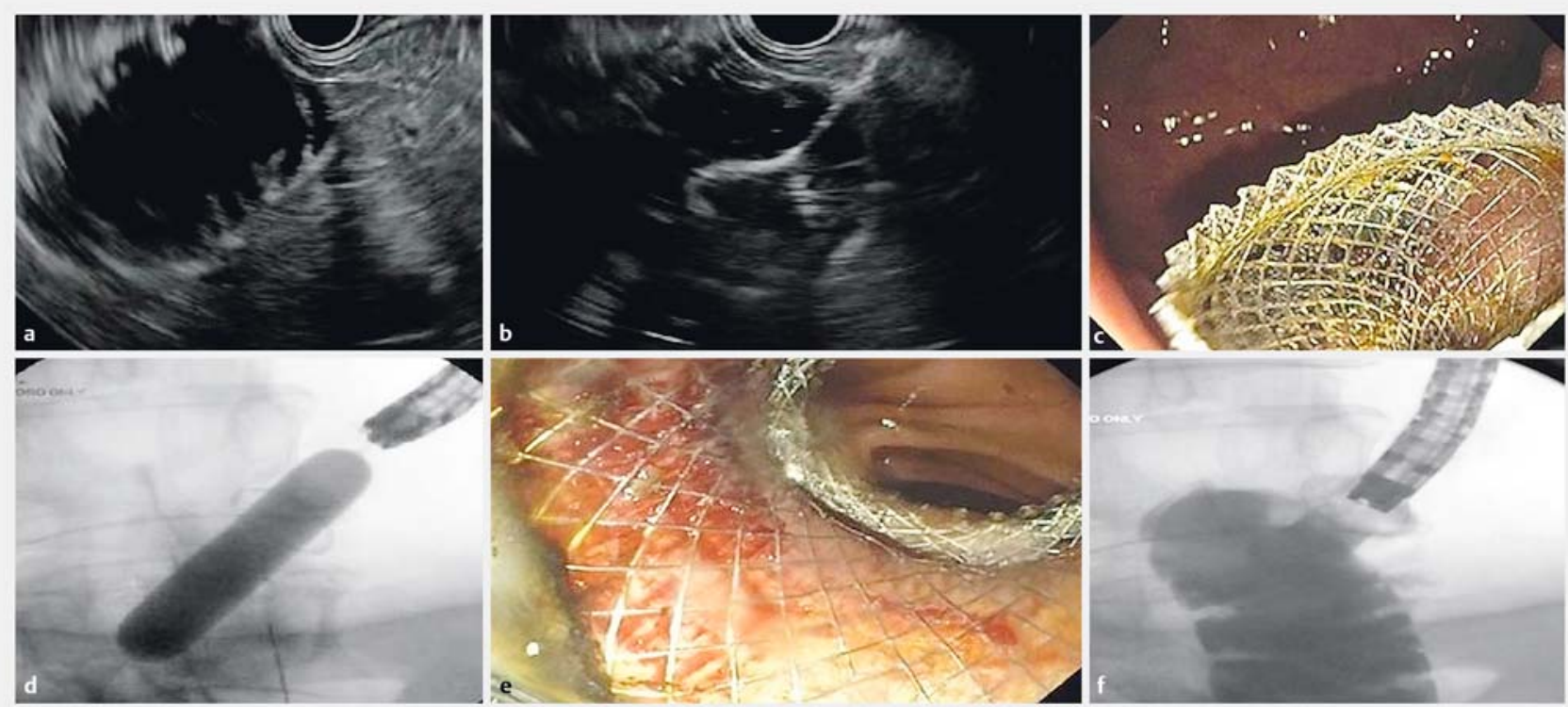

- Fig. 1 EUS-guided gastrojejunostomy using the direct puncture technique. a The small bowel is filled with fluid (saline, contrast and methylene blue) and is easily visualized transgastrically with EUS. It is first punctured with a 19-gauge fine-needle aspiration needle under EUS guidance with aspiration of blue-tinged fluid. Needle is removed and then the loop is directly accessed using the lumen apposing stent system. b The distal anchor flange is deployed first under EUS guidance followed by c deployment of the proximal flange under either endoscopic or sonographic guidance. c Stent is dilated with a balloon to $15 \mathrm{~mm}$ under endoscopic and fluoroscopic guidance. e The jejunum can be seen from within the deployed stent. $\mathbf{f}$ Contrast material is injected and confirms absence of leakage.

modalities. The aim of this study is to compare the clinical success, technical success, adverse events (AEs), length of hospital stay (LOHS) and reintervention rates between EUS-GE and SG].

\section{Patients and methods}

This was a multicenter, international, retrospective comparative study of EUS-GE vs SG] in patients with malignant GOO. The cohort study included consecutive patients who underwent either EUS-GE at 4 centers ( 3 United States, 1 Japan) between January 2013 and August 2015, or SG) at 1 US center between January 2006 and December 2011 for the management of GOO. In the EUS-GE cohort, 23 patients were previously reported by our group in a separate study $[8,13]$. The SG] cohort came from a preexisting database for open gastrojejunostomy.

The study was approved by the Institutional Review Board for Human Research and complied with Health Insurance Portability and Accountability Act (HIPAA) regulations at each of the participating institutions. Inclusion criteria were as follows: (1) documented malignancy; (2) gastric outlet obstruction as defined by symptoms that resulted in decreased oral intake (anorexia, nausea, vomiting, and/or abdominal pain); (3) documentation of obstruction of the gastric outlet endoscopically or radiographically; and (4) EUS-GE or SGJ. Patients who underwent prophylactic SG] during surgical exploration and before development of symptomatic GOO were excluded.

By using electronic medical records, we recorded demographics, type of malignancy, site of obstruction, date of endoscopy or surgery, endoscopy procedural details, evidence of carcinomatosis, need for and type of reinterventions, date of reintervention, and $\mathrm{AEs}$.

\section{EUS-GE procedure}

The procedure was performed as was previously described by Khashab et al. and Itoi et al $[8,13]$. Briefly, patients were given intravenous antibiotics immediately prior to the procedure. EUS-GE was performed using balloon-assisted EUS-GE technique, EUS-guided balloon-occluded gastrojejunostomy bypass (EPASS) or direct puncture technique (. $>$ Fig. 1a-f).

Briefly, the balloon-assisted EUS-GE involves passing a retrieval or dilating balloon catheter over a wire placed in the small bowel, which is then inflated with fluid (contrast/water) while positioned in the duodenum or jejunum distal to the site of obstruction. The fluid-filled balloon is localized transgastrically by EUS and is punctured with a 19-g needle. Bursting of the balloon indicates correct positioning of the needle tip within the small bowel lumen. A guidewire is advanced through the fineneedle aspiration (FNA) needle with subsequent placement of LAMS (cautery tip or regular Axios stent (Xlumena Inc., Mountain View, CA, USA) or Niti-S Spaxus stent (Taewoong Medical Co., Ltd., Ilsan, Korea). Intravenous glucagon injection is given as needed to decrease small bowel peristalsis.

The EPASS approach is performed by first inserting a specialized double balloon enteric tube (Tokyo Medical University Type; Create Medic, Yokohoma, Japan) though the obstruction over a guidewire. Water with contrast is then used to inflate both balloons to anchor and seal the small bowel at two ends. Saline is then infused generously between the 2 balloons. This 
saline insufflation allows for approximation of small bowel loop to the gastric wall leading to easier and safer transgastric EUS puncture and stent insertion with the LAMS.

Lastly, the direct approach entails direct puncture of a small bowel loop adjacent to the gastric wall after initial infusion of fluids into the small bowel. The small bowel is either punctured with a FNA needle followed by advancement of wire and placement of LAMS over the wire or is punctured directly with the cautery tip LAMS.

All patients remained in the hospital after the procedure and antibiotics were continued for 3 days. Liquid diet was commenced the following day and diet was advanced as tolerated. Patients were discharged home when they demonstrated adequate tolerance to oral diet.

\section{Surgical G]}

Patients in the SGJ group underwent an open gastrojejunostomy, either antecolic or retrocolic, while under general anesthesia.

\section{Measured outcomes}

Technical success was defined as adequate positioning and deployment of the stent as determined by the endoscopist and confirmed radiographically. Technical success of SGJ was defined as technical feasibility to perform a gastrojejunostomy. Clinical success or effectiveness of treatment was defined as the ability to tolerate oral intake without vomiting. Recurrent GOO was defined as recurrence of initial symptoms of nausea, vomiting, etc. The need for reintervention (repeat endoscopic or surgical procedures due to recurrent GOO symptoms) was also considered a surrogate for recurrent GOO. The endoscopic and surgical groups were compared in terms of technical success, clinical success, reintervention rates, LOHS, and AEs (infections, perforation, pancreatitis, bleeding, leak, and in-hospital mortality). Severity of AEs was graded according to the ASGE lexicon [14].

\section{Statistical analysis}

Results are reported as mean \pm standard deviation (SD) for quantitative variables, and absolute and relative frequencies for categorical variables. Statistical analyses were performed using the Student's $t$ test and the Mann Whitney $U$ for normally distributed and non-normally continuous variables, respectively. The chi-square or fisher exact test was used to compare categorical variables. Univariable logistic regression analysis was performed to evaluate possible factors associated with clinical success of GOO treatment. Multivariable analysis was performed adjusting for clinically relevant patient characterstics: age, gender, type of intervention (EUS-GE vs SGJ), absence of carcinomatosis, etiology, and site of obstruction. Patient survival time was estimated by the Kaplan-Meier analysis method and the difference between EUS-GE and SGJ groups was tested using Tarone-Ware log rank. Patients were censored at time of analysis or date of last follow up. We also calculated hazard ratios using cox regression adjusting for the same patient characteristics described in the multivariable regression model. A $P$ value $<0.05$ was considered to be statistically significant. All statistical analyses were performed using the SPSS v 21.0 software (SPSS, Chicago, IL, USA).

\section{Results}

A total of 93 patients with malignant GOO were included in the study, who underwent either EUS-GE $(n=30)$ or SGJ $(n=63)$. Pancreatic cancer was the most common cause of malignant GOO in our study ( $n=70,75 \%)$, followed by ampullary cancer.

- Table 1 displays the baselines characteristics stratified according to modality of treatment. Peritoneal carcinomatosis was present in 20 (21\%) patients. The mean duration of follow-up of the entire cohort was 155 days (range 17-688) and was longer in the SG] group (EUS-GE $115 \pm 63$ vs SG] $196 \pm 155$, $P=0.02)$.

\section{EUS-GE cohort}

EUS-GE was attempted in 30 patients with malignant GOO. The mean age was $70 \pm 13.3$ years and $57 \%$ were men. Most common etiology for GOO was pancreatic cancer $(n=17,57 \%)$. Location of GOO was most commonly in the second duodenum $(n=15,50 \%)$ and peritoneal carcinomatosis was present in 13 (43\%) patients. Regarding the techniques of EUS-GE, 22 patients $(73.3 \%)$ underwent EPASS, 6 patients (20\%) underwent balloon assisted GE, and 2 patients underwent direct EUS-GE. The most common type of the stent used was cautery tip axios stent $(70 \%, n=21)$, followed by regular axios stent $(23.3 \%$, $n=7)$, and Spaxus $(6.6 \%, n=2)$. Successful EUS-GJ with placement of a LAMS (technical success) was achieved in 26 (87\%) patients. Four patients had unsuccessful EUS-GE, 2 patients treated with enteral SEMS placements (One patient had stent migration and underwent SGJ) and the other 2 patients underwent SGJ. Clinical success was achieved in all patients who underwent successful LAMS placement ( $87 \%$ of initial cohort). A total of 5 (16\%) AEs occurred and included 3 misdeployment of the stent first flange in the peritoneum and 2 abdominal pain episodes requiring hospitalization. In terms of severity of the AEs, as per the ASGE lexicon, 2 were deemed mild and 3 were severe. There were no fatal AEs. Three patients with stent misdeployment (10\%) were classified as severe AEs based on prolonged hospitalization > 10 days, although all of them were treated conservatively with antibiotics and stent removal. The mean length of hospital stay was 11.6 days (range $2-30$ ). One patient experienced recurrent symptoms of GOO 88 days after the index procedure. Upper endoscopy revealed stent obstruction with food impaction, which was successfully treated with endoscopic extraction.

\section{SG] cohort}

SGJ was attempted in 63 patients with malignant GOO. The mean age was $68 \pm 9.6$ year years and $52 \%$ were men. The most common etiology for GOO was pancreatic cancer $(n=53$, $84 \%)$. Location of GOO was most commonly in the second duodenum $(n=57)$ and peritoneal carcinomatosis was present in 7 (11.12\%) patients. Successful SGJ (technical success) was achieved in all (100\%) patients. Clinical success was achieved in $57(90 \%)$ patients. A total of $16(25 \%)$ adverse events occurr- 
- Table 1 Comparison of baseline patient characteristics of patients undergoing EUS-GE and surgical GJ.

\begin{tabular}{|c|c|c|c|}
\hline & EUS-GE $(n=30)$ & $\begin{array}{l}\text { Surgical G] } \\
(n=63)\end{array}$ & $P$ value \\
\hline Age $(y)$, mean \pm SD & $70 \pm 13.3$ & $68 \pm 9.6$ & 0.8 \\
\hline \multicolumn{4}{|l|}{ Sex, n (\%) } \\
\hline Male & $17(57)$ & $32(52)$ & 0.6 \\
\hline Female & $13(43)$ & $31(49)$ & \\
\hline \multicolumn{4}{|l|}{ Symptoms, n (\%) } \\
\hline Nausea & $23(76)$ & $3555)$ & 0.06 \\
\hline Vomiting & $17(56)$ & $35(55)$ & 1 \\
\hline Abdominal pain & $4(13)$ & $41(65)$ & $<0.001$ \\
\hline Weight loss (Kg) & $28(93)$ & $61(98)$ & 0.6 \\
\hline \multicolumn{4}{|l|}{ Etiology, $\mathrm{n}(\%)$} \\
\hline Gastric cancer & $5(17.6)$ & $0(0)$ & 0.002 \\
\hline Ampullary cancer & $2(6.7)$ & $9(14)$ & 0.49 \\
\hline Duodenal cancer & $0(0)$ & $1(1.5)$ & 0.9 \\
\hline Pancreatic cancer & $17(56)$ & $53(84.5)$ & 0.003 \\
\hline Biliary/gallbladder cancer & $2(6.7)$ & $0(0)$ & 0.1 \\
\hline Extrinsic/metastatic cancer & $4(13)$ & $0(0)$ & 0.009 \\
\hline \multicolumn{4}{|l|}{ Site of the obstruction, $\mathbf{n}(\%)$} \\
\hline Pyloric/duodena bulb & $7(23)$ & $2(3)$ & 0.004 \\
\hline Second part of the duodenum & $15(50)$ & $57(90)$ & $<0.001$ \\
\hline Distal duodenum/proximal jejunum & $8(27)$ & $4(7)$ & 0.01 \\
\hline Peritoneal Carcinomatosis, n (\%) & $13(43)$ & $7(11)$ & $<0.001$ \\
\hline
\end{tabular}

ed and included infection in 8 patients, anastomotic leak in 4, postsurgical persistent ileus in 1 , agitation/delirium in 2 , and pulmonary embolism in 1 . A total of $13 \mathrm{AEs}$ were rated as mild, 3 as moderate, and 0 as severe. There were no fatal AEs. The mean length of hospital stay was 12 days (range $3-39$ ). A total of $9(14 \%)$ patients experienced recurrent symptoms of GOO at a mean of 121 days (range 17 -154) after the index procedure. Upper endoscopy was performed only in 5 patients and revealed a stricture of the afferent limb or kink in the efferent limb in 4 patients and suggested delayed gastric emptying without obstruction in 1 patient (balloon dilation of the G) in 3 patients, enteral stent placement in 1 patient, and Peg J placement in 1 patient).

\section{Comparison of EUS-GE and SG]}

Baseline characteristics between the 2 groups were equivalent except for the etiology of GOO, site of the obstructions and the rate of peritoneal carcinomatosis. The rates of pancreatic cancer, gastric cancer and extrinsic/metastatic cancer were higher in the EUS-GE group. Patients in SG] more frequently presented with abdominal pain (41 vs $4, P \leq 0.001$ ) ( T Table 1 ). The clinical outcomes of the two groups are summarized in $>$ Table 2 .
Although the technical success rate was significantly higher in the SGJ group as compared to the EUS-GE group (100\% vs. $87 \%$, OR 3.4, $P=0.009)$, the clinical success rate was similar between both groups ( $90 \%$ vs. $87 \%, P=0.18$, OR $1.7,95 \% \mathrm{Cl}: 0.44-$ 7.07). Univariable and multivariable analyses showed that both techniques were not different in terms clinical success. The only independent predictor of clinical success was absence of peritoneal carcinomatosis ( $\$$ Table 3 ). The rate of AEs was lower in the EUS-GE group, but the difference was not statistically significant ( $16 \%$ vs $25 \%, p=0.3$ ). The mean LOHS was also similar in the EUS-GE group compared to SG) (11.6 days \pm 6.6 vs. 12 days \pm 8.2 days, $P=0.35)$. The rate of GOO recurrence was not different between the two groups ( $3 \%$ vs. $14 \%, P=0.08$ ). Similarly, the mean time to reintervention was similar ( 88 days vs. 121 days, $P=0.83$ ). Despite survival being longer for EUS in the surgical arm (log rank test, $p=0.006$ ) ( $>$ Figure 2 ), the hazard ratio was not significant when other relevant variables were considered (HR 1.5, $95 \% \mathrm{Cl} 0.8-2.9, P=0.18$ ) ( $>$ Table 4). 
- Table 2 Outcome comparisons of patients who underwent EUS-GE or surgical G] for malignant gastric outlet obstruction.

\begin{tabular}{|c|c|c|c|}
\hline & EUS-GE $(n=30)$ & Surgical GJ $(n=63)$ & OR (95\%Cl), P value \\
\hline Technical success, n (\%) & $26(87)$ & $63(100)$ & $0.009^{*}$ \\
\hline Clinical success, $n(\%)$ & $26(87)$ & $57(90)$ & $0.8(0.44-7.07), 0.18$ \\
\hline Recurrent GOO, n (\%) & $1(3)$ & $9(14)$ & $0.2(0.02-1.45), 0.08$ \\
\hline Adverse events, $\mathrm{n}(\%)$ & $5(16)$ & $16(25)$ & $0.3(0.19-1.79), 0.3$ \\
\hline Mean length of hospitalization (days), mean \pm SD & $11.6 \pm 6.6$ & $12 \pm 8.2$ & $0.35^{*}$ \\
\hline
\end{tabular}

- Table 3 Univariable and multivariable analysis of predictors of clinical success.

\begin{tabular}{|l|l|l|}
\hline Univariable analysis & $\begin{array}{l}\text { Clinical success, } \\
\text { OR (95\%CI) }\end{array}$ & P value \\
\hline $\begin{array}{l}\text { Modality (EUS-GEI } \\
\text { Surgical GJ) }\end{array}$ & $0.8(0.44-7.07)$ & 0.18 \\
\hline Age & $0.9(0.94-1.05)$ & 0.95 \\
\hline Gender (M/F) & $0.6(0.16-2.81)$ & 0.59 \\
\hline Absence of carcinomatosis & $19(3.61-103.88)$ & 0.001 \\
\hline $\begin{array}{l}\text { Site of obstruction (Distal } \\
\text { vs. others) }\end{array}$ & $1(0.12-10.05)$ & 0.91 \\
\hline $\begin{array}{l}\text { Etiology (pancreatic cancer } \\
\text { vs others) }\end{array}$ & $1(0.23-4.01)$ & 0.95 \\
\hline $\begin{array}{l}\text { Modality (EUS-GE/ } \\
\text { Surgical G]) }\end{array}$ & $0.1(0.85-77.76)$ & 0.06 \\
\hline Age & $0.9(0.92-1.07)$ & 0.89 \\
\hline \begin{tabular}{l} 
Gender \\
\hline Absence of carcinomatosis
\end{tabular} & $0.2(0.03-2.38)$ & 0.24 \\
\hline $\begin{array}{l}\text { Site of obstruction (distal } \\
\text { vs others) }\end{array}$ & $3.7(0.19-73.15)$ & 0.38 \\
\hline $\begin{array}{l}\text { Etiology (pancreatic } \\
\text { cancer vs others) }\end{array}$ & $1.5(0.19-11.68)$ & 0.7 \\
\hline
\end{tabular}

\section{Discussion}

Management of malignant GOO has changed in recent years with the widespread availability and ease of placement of enteral SEMS [15]. Currently, most of these patients are managed endoscopically as surgery is considered invasive, especially in these often terminally sick patients [7]. Nonetheless, the optimal treatment modality continues to be controversial especially in view of mixed results from 3 randomized trials comparing enteral SEMS to SC], with 2 trials favoring enteral SEMS [4,5] and 1 favoring SG] [6]. Overall, SEMS is primarily offered to these patients as a minimally invasive palliative modality with the understanding that recurrent GOO due to tumor ingrowth/ overgrowth is a common phenomenon [7]. The advantage of $\mathrm{SC}$ ] is long-lasting palliation with low risk of symptom recur-
- Table 4 Hazard ratios from Cox regression analysis

\begin{tabular}{|l|l|l|}
\hline Variable & HR $(\mathbf{9 5} \% \mathbf{C l})$ & $\boldsymbol{P}$ value \\
\hline EUS-GE vs SGJ & $1.5(0.8-2.9)$ & 0.18 \\
\hline Pancreatic cancer vs others & $1.6(0.89-2.95)$ & 0.11 \\
\hline $\begin{array}{l}\text { Site of obstruction (distal } \\
\text { vs others) }\end{array}$ & $1.2(0.50-2.92)$ & 0.67 \\
\hline Absence of carcinomatosis & $0.73(0.38-1.38)$ & 0.33 \\
\hline Gender (M/F) & $0.74(0.44-1.25)$ & 0.27 \\
\hline Age & $1.0(0.98-1.02)$ & 0.63 \\
\hline
\end{tabular}

rence [6]. However, surgical complications are not uncommon and include delayed gastric emptying, prolonged hospital stay, increased costs, and delay in cancer treatment [7].

Advances in interventional EUS techniques coupled with recent development of LAMS have rendered EUS-GE feasible [16]. In principle, EUS-GE offers the advantage of enteral stenting due to its minimally invasive nature. At the same time, it potentially offers long-lasting symptom palliation similar to that offered by SG] as the LAMS is placed away from the site of malignant luminal obstruction without risk of tumor ingrowth/overgrowth [8]. Comparative trials are needed to confirm these assumptions. The current study is the first to compare outcomes of EUS-GE to that of SG] in patients with malignant GOO. A total of 93 patients were studied and the rate of clinical success was similar between both groups ( $92 \%$ vs. $87 \%, P=0.42$ ). There was no significant difference in the rate of AEs between both groups ( $16 \%$ vs $25 \%, P=0.3$ ). Importantly, the rate of GOO recurrence was equally low in both groups ( $3 \%$ vs. $14 \%, P=0.08$ ), which suggests that EUS-GE provides long-lasting symptom palliation of GOO similar to that of surgical bypass. The duration of follow-up was, however, longer in the surgical arm, possibly due to longer survival. Type of intervention (EUS-GE or SGJ) did not affect survival when other relevant variables were factored in ( $>$ Table 4).

Outcomes of patients who underwent EUS-GE are in line with recently reported results. Khashab et al. reported on the first US clinical experience with EUS-GE in 10 patients with benign $(n=7)$ or malignant GOO $(n=3)$ [11]. Technical success oc- 


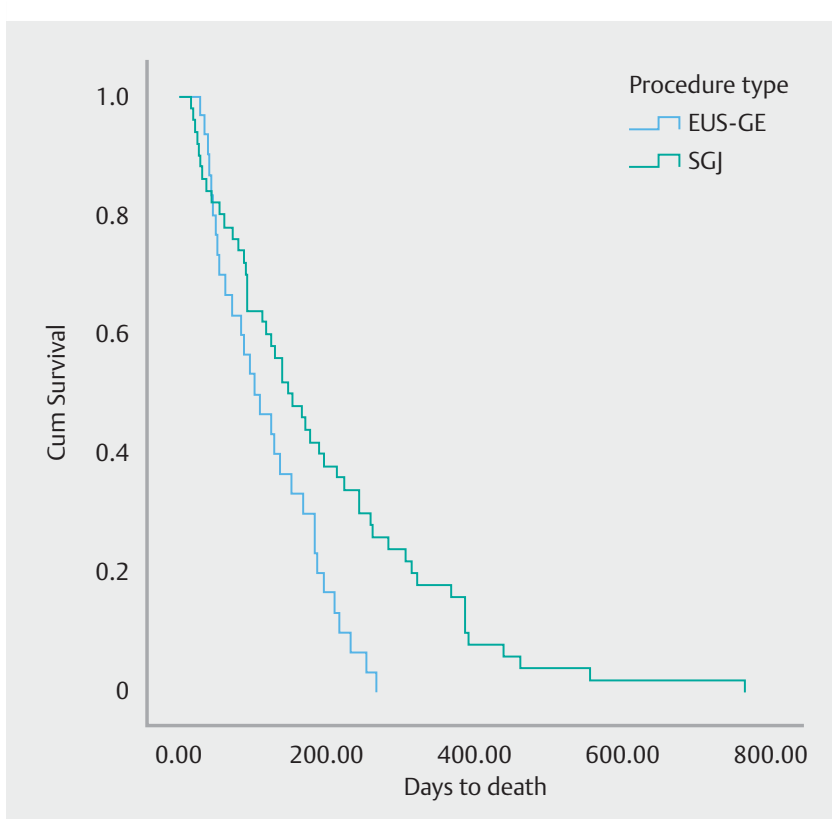

- Fig. 2 Kaplan Meier plot estimates the overall survival for patients undergoing EUS-GE vs SGJ. EUS-GE: Median survival 103 days with $95 \% \mathrm{Cl} 45-160$. SG]: Median survival 148 days with $95 \% \mathrm{Cl}$ $100-195$. Median overall survival for the entire cohort is 130 days with $95 \% \mathrm{Cl} 97-162$. There was a significant difference in survival times between the 2 groups ( $P=0.02$ using Tarone-Ware; $P=0.006$ using log rank)

curred in 9 patients (90\%). There were no procedure-related AEs. Clinical success with resumption of solid oral intake was achieved in all 9 patients (100\%) who underwent successful EUS-GE. There was no symptom recurrence during a mean follow-up period of 150 days [11]. Tyberg et al. studied 26 patients with GOO who underwent EUS-GE [12]. Technical success was achieved in 24 patients (92\%) and clinical success in 22 patients ( $85 \%)$. AEs, including peritonitis, bleeding, and surgery, occurred in 3 patients (11.5\%). One death was reported due to peritonitis.

Despite the impressive results suggested both by the current study and the limited available literature, EUS-GE remains a challenging procedure and requires expertise in interventional EUS. The EUS-GE technique has not been standardized yet. The small bowel is a mobile intraperitoneal organ and can move away from the gastric wall during the procedure. In addition, the small bowel may be pushed away from the stomach while advancing the wire through the FNA needle after small bowel puncture. The "throw" of the sheath of currently available LAMS is relatively short $(8 \mathrm{~cm})$ and it may not be feasible to consistently reach an adjacent small bowel loop. The balloon-assisted EUS-GE technique used in the current study is crucial to ensure that a loop of small bowel rather than the coIon is accessed. Advancement of the balloon catheter may be challenging at times due to looping in the stomach. Use of a stiff guidewire and sometimes a long overtube are helpful for overcoming this technical challenge. Small bowel section around the ligament of Treitz is the part typically adjacent to the stomach. Therefore, management of GOO localized to this area may render the procedure more challenging and sometimes not possible. Lastly, currently available LAMS are relatively small in diameter $(10 \mathrm{~mm}$ and $15 \mathrm{~mm})$; in addition, resheathing of the stent is not currently possible.

The current study has multiple limitations. It was a retrospective comparative trial with inherent limitations due to its design. The non-randomized nature of the study may introduce bias. Consecutively treated patients were included to limit selection bias. Patients in the SGJ group were recruited from one center, while patients in the EUS-GE group were recruited from multiple centers. This may have introduced heterogeneity to the study population. All surgical and endoscopic procedures were performed at tertiary centers and thus, results may not be generalizable. All patients in the surgical group underwent open surgery which may not be the standard of care at some centers. Lastly, the majority of EUS-GE procedures were facilitated using the double-balloon device [13]. This device is not widely available yet.

\section{Conclusions}

In conclusion, EUS-GE is associated with equivalent efficacy and safety as compared to surgical GJ. This is the first comparative trial between both techniques and suggests EUS-GE as a noninferior but less invasive alternative to surgery. A prospective comparative trial is needed to confirm these intriguing results.

\section{Competing interests}

Mouen Khashab is a consultant for Boston Scientific. Todd Baron is a consultant for Boston Scientific. Jose Nieto is a consultant for Boston Scientific. Takao Itoi is a consultant for Boston Scientific

\section{References}

[1] van Heek NT, van Geenen RC, Busch OR et al. Palliative treatment in "peri"-pancreatic carcinoma: stenting or surgical therapy? Acta Gastro-enterologica Belgica 2002; 65: 171 - 175

[2] van Hooft JE, Uitdehaag MJ, Bruno MJ et al. Efficacy and safety of the new WallFlex enteral stent in palliative treatment of malignant gastric outlet obstruction (DUOFLEX study): a prospective multicenter study. Gastrointest Endosc 2009; 69: 1059-1066

[3] Johnsson E, Thune A, Liedman B. Palliation of malignant gastroduodenal obstruction with open surgical bypass or endoscopic stenting: clinical outcome and health economic evaluation. World J Surgery 2004; 28: 812-817

[4] Mehta S, Hindmarsh A, Cheong E et al. Prospective randomized trial of laparoscopic gastrojejunostomy versus duodenal stenting for malignant gastric outflow obstruction. Surg Endosc 2006; 20: 239-242

[5] Fiori E, Lamazza A, Volpino P et al. Palliative management of malignant antro-pyloric strictures. Gastroenterostomy vs. endoscopic stenting. A randomized prospective trial. Anticancer Res 2004; 24: $269-271$ 
[6] Jeurnink SM, Steyerberg EW, van Hooft JE et al. Surgical gastrojejunostomy or endoscopic stent placement for the palliation of malignant gastric outlet obstruction (SUSTENT study): a multicenter randomized trial. Gastrointest Endosc 2010; 71: 490-499

[7] Khashab M, Alawad AS, Shin E] et al. Enteral stenting versus gastrojejunostomy for palliation of malignant gastric outlet obstruction. Surg Endoscop 2013; 27: 2068 - 2075

[8] Khashab MA, Baron TH, Binmoeller KF et al. EUS-guided gastroenterostomy: a new promising technique in evolution. Gastrointest Endosc 2015; 81: $1234-1236$

[9] Ngamruengphong S, Kumbhari V, Tieu AH et al. A novel "balloon/ snare apparatus" technique to facilitate easy creation of fistula tract during EUS-guided gastroenterostomy. Gastrointest Endosc 2016: DOI: 10.1016/j.gie.2016.03.1493

[10] Binmoeller KF, Shah J. A novel lumen-apposing stent for transluminal drainage of nonadherent extraintestinal fluid collections. Endoscopy 2011; 43: $337-342$

[11] Khashab MA, Kumbhari V, Grimm IS et al. EUS-guided gastroenterostomy: the first U.S. clinical experience (with video). Gastrointest Endosc 2015; 82: 932 - 938
[12] Tyberg A, Perez-Miranda M, Sanchez-Ocana R et al. Endoscopic ultrasound-guided gastrojejunostomy with a lumen-apposing metal stent: a multicenter, international experience. Endosc Int Open 2016; 4: E276-E281

[13] Itoi T, Ishii K, Ikeuchi N et al. Prospective evaluation of endoscopic ultrasonography-guided double-balloon-occluded gastrojejunostomy bypass (EPASS) for malignant gastric outlet obstruction. Gut 2016; 65: $193-195$

[14] Cotton PB, Eisen GM, Aabakken L et al. A lexicon for endoscopic adverse events: report of an ASGE workshop. Gastrointest Endosc 2010; 71: $446-454$

[15] Adler DG, Baron TH. Endoscopic palliation of malignant gastric outlet obstruction using self-expanding metal stents: experience in $36 \mathrm{pa}-$ tients. Am J Gastroenterol 2002; 97: $72-78$

[16] Binmoeller KF, Shah JN. Endoscopic ultrasound-guided gastroenterostomy using novel tools designed for transluminal therapy: a porcine study. Endoscopy 2012; 44: 499-503 